\title{
The Prevalence of Isolated Neutropenia at High Altitude in Southern Saudi Arabia: Does Altitude Affect Leucocyte Count?
}

This article was published in the following Dove Press journal: International Journal of General Medicine

\author{
Husain Y Alkhaldy (D) 1,2 \\ Zuhier A. Awan 3,4 \\ Ahmed A Abouzaid ${ }^{4,5}$ \\ Hossameldin M Elbahaie ${ }^{4,6}$ \\ Saeed M Al Amoudi ${ }^{4}$ \\ Mohamed Andarawi ${ }^{7}$ \\ Shehata F Shehata ${ }^{8,9}$ \\ 'Department of Internal Medicine, \\ College of Medicine, King Khalid \\ University, Abha, Saudi Arabia; ${ }^{2}$ Research \\ Center for Advanced Materials Science, \\ King Khalid University, Abha, Saudi \\ Arabia; ${ }^{3}$ Department of Clinical \\ Biochemistry, Faculty of Medicine, King \\ Abdulaziz University, Jeddah, Saudi \\ Arabia; ${ }^{4}$ Department of Clinical \\ Pathology, Al-Borg Medical Laboratories, \\ Jeddah, Saudi Arabia; ${ }^{5}$ Department of \\ Medical Biochemistry, Faculty of \\ Medicine, Al-Azhar University, Cairo, \\ Egypt; ${ }^{6}$ Clinical Pathology Department, \\ Suez Canal University, Ismailia, Egypt; \\ ${ }^{7}$ Department of Pathology, College of \\ Medicine, King Khalid University, Abha, \\ Saudi Arabia; ${ }^{8}$ Department of \\ Community Medicine, College of \\ Medicine, King Khalid University, Abha, \\ Saudi Arabia; ${ }^{9}$ High Institute of Public \\ Health, Alexandria University, Alexandria, \\ Egypt
}

Correspondence: Husain Y Alkhaldy Department of Internal Medicine, College of Medicine, King Khalid University, Abha, P.O.Box 64I, Aseer 6142I, Saudi Arabia Tel +96672417578

Fax +966 724l8III

Email halkhaldy@kku.edu.sa
Introduction: Isolated neutropenia is a common problem in hematology practice. Benign ethnic neutropenia (BEN) is the common form of neutropenia worldwide and it affects mainly African and Middle Eastern ethnicities. Most cases of isolated mild and moderate neutropenia are benign and associated with no clinical significance. The aim of this study was to estimate the prevalence of isolated neutropenia at high-altitude southern Saudi Arabia. Methods: A large dataset of complete blood counts (CBCs) was analyzed for walk-in patients of both genders, of age ranges from 12 to 60 years doing blood tests at a commercial lab for high altitude - 2,270 meters above sea level - (HA) group, and from sea level SL group. Abnormal biochemical or CBC results were excluded before analysis.

Results: For HA group, $3123 \mathrm{CBCs}$ were analyzed and for SL group 18,427 CBCs were analyzed. The prevalence of mild neutropenia, defined as absolute neutrophil count (ANC) in the range of $1.0-.5 \times 10^{9} / \mathrm{L}$, was $12.4 \%(\mathrm{n}=399)$ versus $5.6 \%(\mathrm{n}=1025)$, while moderate neutropenia, defined as ANC of $0.5-1 \times 10^{9} / \mathrm{L}$, was present $6 \%(\mathrm{n}=191)$ versus $1.45 \%$ $(n=269)$ in the high altitude and sea level groups, respectively. Severe neutropenia, defined as neutrophil count less than $0.5-1 \times 10^{9} / \mathrm{L}$, was rare in both groups.

Conclusion: Isolated neutropenia is common in Saudis living at high altitude. While benign ethnic neutropenia (BEN) is thought to be the major contributing factor to this high prevalence, other factors including environmental factors and altitude are possible contributing factors along with underlying ethnic neutropenia.

Keywords: ethnic neutropenia, high altitude, hematological, leukocyte count

\section{Introduction}

Isolated neutropenia, in the absence of other hematological abnormalities, is a common problem in the primary care and consultation settings of the hematology practice. ${ }^{1,2}$ There are many causes of neutropenia, whether congenital or acquired. ${ }^{3}$ Work-up of neutropenia is usually dictated by the associated clinical scenario but when chronic, stable, and mild or moderate in nature, isolated neutropenia is usually a benign condition with no increased risk of infection or progression to hematological malignancies.

Benign Ethnic neutropenia (BEN) - also known as chronic benign neutropenia or constitutional neutropenia - is the most common form of isolated neutropenia worldwide. First described in 1941, in African Americans, this genetically determined condition affects many non-Caucasian ethnicities including African and Middle Eastern ethnicities. ${ }^{4}$ BEN is usually associated with a mild to moderate 
decrease in the absolute neutrophil count (ANC) that is associated with no clinical significance, and no increased risk of infection. The genetic basis of benign ethnic neutropenia has been recently defined and the long-term excellent prognosis is well described. ${ }^{5,6}$

In susceptible ethnicities, a variable benign ethnic neutropenia prevalence of $25-50 \%$ has generally been reported. ${ }^{1}$ Arab ethnicity is among those who constitutionally harbor this ethnic neutropenia. ${ }^{7}$ In Saudi Arabia, the prevalence of benign ethnic neutropenia is not definitely known. One study estimated the prevalence of BEN in Saudi Arabia at $20 \%{ }^{8}$ In clinical practice, assigning the diagnosis of benign ethnic neutropenia would usually mean thorough evaluation to exclude many other etiologies including congenital and acquired neutropenia. This usually entails an extensive work-up including sometimes invasive testing like bone marrow aspiration and biopsy, causing unnecessary worries for the patients. When such a health problem is categorized as relatively common and associated with no increased risk, one might think that this should be accepted as a normal variation in susceptible ethnicity which in turn leads to offering no more workups.

The effect of high altitude on hemoglobin concentration, red blood cell counts, and their indices are well established. ${ }^{9,10}$ However, little is known about the effect of high altitude on white blood counts (leucocytes). In our practice, mild and moderate neutropenia is relatively common, and the work-up is usually unrevealing. To our knowledge, there have been no large epidemiological studies to estimate the normal range of blood counts in our high-altitude area (Abha city located at 2270 meters above sea level in Aseer Region, southern Saudi Arabia). Hence, we conducted this study to determine a more precise estimation of the prevalence of benign ethnic neutropenia in high altitude in southern Saudi Arabia.

\section{Methods}

This study was designed and conducted in accordance with the Declaration of Helsinki principles. The study protocol and study subject consent waiver were approved by the ethical and research committee of King Khalid University, Abha, Saudi Arabia. (ECM\#2020-0905) - (HAPO-06-B-001).

In this retrospective study, CBC results were screened over a year (2018-2019) for self-referred walk-in people to do a health checkup or routine blood tests at Alborg laboratory, Abha, Aseer region (moderately high altitude ranging from $2270 \mathrm{~m}$ to $3000 \mathrm{~m}$ above sea level). Lab tests were screened from Platinum package commonly requested for comprehensive blood check (includes $\mathrm{CBC}$, renal profile; serum blood urea nitrogen, creatinine, liver profile; ALT, AST, total bilirubin, direct bilirubin, lipid profile; cholesterol, HDL, LDL, triglyceride, thyroid function tests; TSH, $\mathrm{T} 4$, and a fasting blood sugar). Customers usually answered a pre-test questionnaire and have their blood pressure checked. A control sea level sample of $\mathrm{CBC}$ results was also collected from another Alborg laboratory at Jeddah, a large coastal city in western Saudi Arabia.

\section{Inclusion and Exclusion Criteria}

People of both genders of age from 12 to 60 years were included. Most of the participants in high altitude cohort $(>95 \%)$ are Saudis. To estimate the WBCs reference range, the following exclusion criteria were applied. Any significant abnormalities in the biochemical, kidney, liver, or lipid profile were excluded. Hb less than $11 \mathrm{~g} / \mathrm{dl}$ or more than $19 \mathrm{~g} / \mathrm{dl}$ was excluded. Thrombocytopenia $\left(<150 \times 10^{9} /\right.$ L) or platelets count more than $450 \times 10^{9} / \mathrm{L}$ was excluded. WBCs more than $11 \times 10^{9} / \mathrm{L}$ were excluded. For the low WBCs, the exclusion was applied only if other cell lines were affected, ie concurrent anemia or thrombocytopenia.

For the sea level group, CBCs only were reviewed, and exclusion criteria per abnormality in CBCs per the above criteria were applied. (Figure S1-S2)

\section{Blood Collection and Laboratory Analysis}

For CBCs, venous blood samples were collected into liquid K3EDTA tubes and were processed within 2 hours of collection. Sysmex automated analyzers (Sysmex corporation, Kobe, Japan) was used for WBCs enumeration and differential count.

\section{Data Analysis}

The participants' data were de-identified for any personal information before importing to excel datasheet. After data were extracted, they were revised, coded, and fed into statistical software IBM SPSS version 22 (SPSS, Inc. Chicago, IL). All statistical analysis was done using two-tailed tests. Normal distribution assessment was applied for all scale parameters. All CBC parameters descriptive analysis using trimmed mean with its $95 \%$ confidence interval, median, range, Standard deviation (SD) and percentiles (2.5th and 97.5 th) were used to assess the normal population range. Any extreme value was minorized using a larger value 


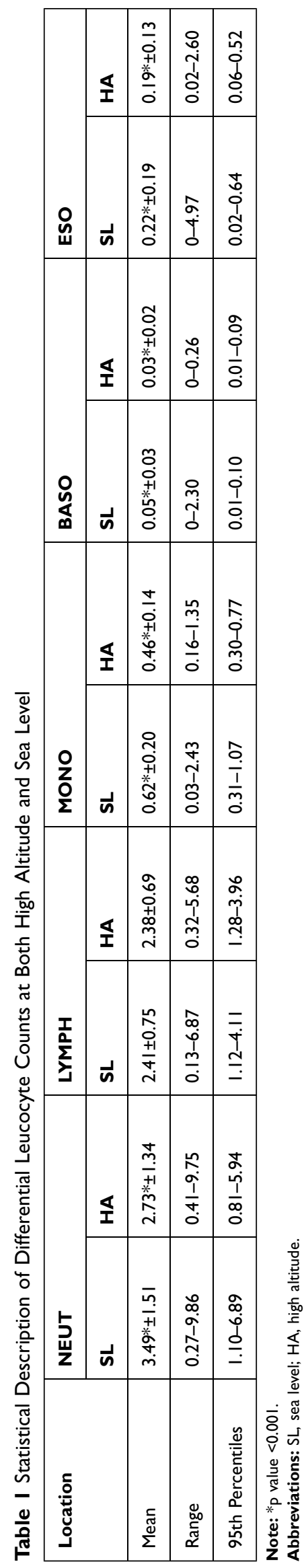

Table 2 Statistical Characteristics of Absolute Neutrophil Counts and Prevalence of Neutropenia at High Altitude and at Sea Level

\begin{tabular}{|l|l|l|}
\hline & High Altitude & Sea Level \\
\hline $\mathrm{N}$ & 3213 & 18,427 \\
\hline Mean (SD) & $2.73 \pm 1.34 *$ & $3.49 \pm 1.5 \mathrm{I}^{*}$ \\
\hline 95 th percentile range & $0.81-5.94$ & $1.10-6.89$ \\
\hline ANC $<2 \times 10^{9} / \mathrm{L}$ & $1077(33.5 \%)$ & $3077(16.7 \%)$ \\
\hline ANC $<1.5 \times 10^{9} / \mathrm{L}$ & $594(18.5 \%)$ & $1308(7.1 \%)$ \\
\hline ANC $<1.0 \times 10^{9} / \mathrm{L}$ & $193(6 \%)$ & $276(2 \%)$ \\
\hline ANC $<0.5 \times 10^{9} / \mathrm{L}$ & 2 & 7 \\
\hline
\end{tabular}

Note: *p value $<0.001$.

winsorization method. Differences between variables were considered significant if $95 \%$ confidence interval did not show overlap. Any significant difference in the WBCs subset between the two groups and/or with gender or age group was tested using two-tail Student's $t$-test. Significance level was set at $(<0.05)$

\section{Results}

In total, 4180 and 27,000 samples were screened for primary and control groups, respectively (Figure S3). After applying exclusion criteria, 3123 samples were selected for high-altitude group, and 18,427 samples from sea level group. The descriptive analysis for the leucocyte count is demonstrated in Table 1, while prevalence and severity of neutropenia is depicted in (Table 2)

The mean neutrophil count for the high-altitude group was $2.7 \times 10^{9} / \mathrm{L}$ (95\% CI 2.68-2.77), and the median was 2.51 (SD 1.356). That is significantly different ( $p$ value $<0.01$ ) compared to the sea level group with a mean ANC of $3.49(95 \% \mathrm{CI}$ 3.47-3.51) and median of 3.31 (SD1.51) (as shown in Table 2). The difference remained significant when comparing the HA and SL per age group. No gender or age difference was demonstrated within the high altitude and sea level. The rest of CBC parameters for both sea level and high altitude were depicted in Supplement 1 (Table 3)

The overall prevalence of isolated neutropenia at high altitude is $18.5 \%(\mathrm{n}=594)$, mainly mild $12.4 \%(\mathrm{n}=399)$ while the prevalence of moderate neutropenia is $6 \%(\mathrm{n}=191)$. At the sea level, the overall prevalence of isolated neutropenia is $7.1 \%(\mathrm{n}=1308)$, with individuals having mild neutropenia $5.5 \%(\mathrm{n}=1025)$ and moderate neutropenia at $2 \%(\mathrm{n}=276)$. Severe neutropenia defined as ANC less than $0.5 \times 10^{9} / \mathrm{L}$ 


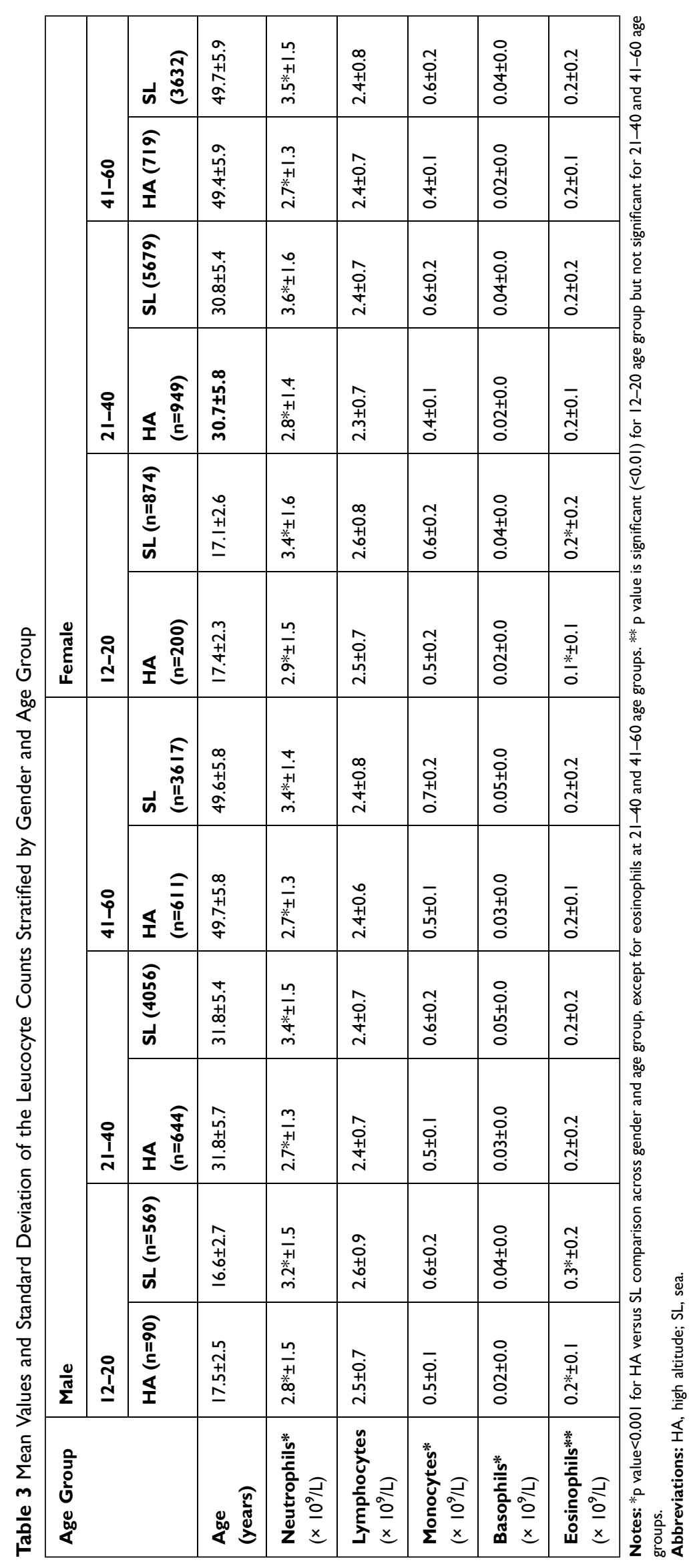


was rare in both groups ( 2 persons in the high altitude and 7 persons in the sea level group).

\section{Discussion}

In this retrospective study, using a large dataset of $\mathrm{CBC}$ results of walk-in patients at high altitude, we found that the mean neutrophils fraction and the 95 th percentile range are lower in Saudis residing at high altitude compared to the currently used reference range. The overall prevalence of isolated neutropenia at high altitude is $18.5 \%(n=594)$, mainly mild $12.4 \%(n=399)$ while the prevalence of moderate neutropenia is $6 \%(n=191)$. We postulate that this isolated neutropenia is most likely BEN. In a study in Saudi Arabia, the prevalence of isolated neutropenia was found to be $20 \%{ }^{8}$ BEN has been previously reported in Arabs and Middle Eastern populations. ${ }^{11-13}$

The prevalence of isolated neutropenia between individuals at high altitude and sea level showed a significant difference. In fact, other fractions of the white blood counts, apart from the lymphocytes fraction are statistically different (lower) in the high-altitude group compared to sea level group. While most participants from high altitude are Saudi, the ethnicity was not directly assessed in the sea level group. Jeddah is a large multinational city - with non-Saudi residents constituting about 50\% of its population. The difference in the sample size might also affect the results. Nonetheless, the effect of high altitude on the WBCs count, mainly neutrophils, should be further explored. Basically, the effect of high altitude on leukocytes count is thought to be less pronounced compared to hemoglobin, red blood counts, and their indices. Many studies indicated that newborn infants born at higher altitudes have higher neutrophils count compared to those born at sea level, ${ }^{14-16}$ but such an effect was not reported in adults. A study reported a mild but statistically significant drop in the neutrophil counts after 8 months of continuous exposure to $3500 \mathrm{~m}$ of altitude. ${ }^{17}$ Old literature indicates that neutrophil counts decreased with altitude. ${ }^{18}$ However, we found no recent literature to agree with these findings in the era of automated analyzers. Most of the reviewed literature focused on the change in the $\mathrm{RBC}$ indices, but very few on the white blood count. On the other hand, at moderately high altitude $(1700 \mathrm{~m}$ above sea level) in China, the mean ANC count in highlanders was $3.6 \pm 1.3$ (comparable to our sea level group). ${ }^{19}$ Our study also showed a statistically significant decrease, though might not be clinically significant, in the monocytic, all granulocytic but not lymphocytic fractions which suggest a true high-altitude effect. One may postulate that the expanded erythroid activity in response to altitude associated hypoxia is accompanied by relative decrease activity of the myeloid/monocytic lineage. Another theory is that plasma volume change associated with high-altitude might explain this change if coupled with relative lymphocytosis. Further investigation is needed to confirm or refute this hypothesis.

It is established that BEN is linked to the genetic deletion of the Duffy antigen receptor for chemokines (DARC-null genotype), which arises from a single nucleotide polymorphism, is thought to offer a selective advantage against the invasion of red blood cells by plasmodium vivax malaria. ${ }^{6,11,20,21}$ Some researchers suggest that the absence of Duffy antigen in blood bank serology grouping can be used to screen the ethnic neutropenia. ${ }^{22}$ If confirmed, this will offer a simple way to secure a diagnosis of BEN in the appropriate clinical setting without the need for extensive testing.

In the primary care setting, non-specialist practitioners view neutropenia as life-threatening conditions; however, when isolated, benign causes of neutropenia are more common. $^{23,24}$ Direct bone marrow examinations in individuals with BEN show normal cellularity of the bone marrow and leukocyte maturation. ${ }^{25}$ The long-term prognosis is excellent and increased infection does not seem to be a risk. $^{5,26}$ It is extremely unlikely for chronic isolated neutropenia to progress to sinister diagnoses like hematological malignancies. ${ }^{27}$

The finding of this study should provide an insight into the practicing haematologists about the prevalence and magnitude of the benign ethnic neutropenia in our population. Ideally, a long-term follow-up is needed to confirm the good prognosis of the condition in our population. Further studies of prospective nature are needed to explore the need for adjusting the reference range for the studied population. A lower reference range might result in misclassification, especially among the non-specialists' practitioners, of other neutropenia, with a potential underlying serious diagnosis with the more common benign neutropenia. Currently, the diagnosis remains that of exclusion and the work-up should be wisely individualized especially in those in the mild to moderate spectrum. Clinical assessment, chronicity of the problem, and other associated manifestations are important factors in the evaluation of neutropenia. ${ }^{28,29}$

Nonetheless, the current study still has some limitations. For instance, detailed demographic characterization 
including tribal origin was not available. Whether participants from high-altitude group are long-term dwellers or recent arrival was not assessed. The sea level group members may not always match the ethnicity criteria. Moreover, analytic factors that affect the white blood counts, eg smoking, exercise, stress, and timing of sampling were not assessed.

\section{Conclusion}

Isolated neutropenia is common in Saudis residing at high altitude. The most likely underlying etiology is BEN. Hematologists should wisely consider the needed work-up for patients presenting with otherwise isolated neutropenia. In the appropriate clinical settings, simple evaluation might identify BEN and obviate the need for extensive work-ups.

The current study also shows a possible effect of high altitude on the myeloid leucocytes and monocytes, which needs further investigation.

\section{Data Sharing Statement}

Data used in this research are available to readers upon request.

\section{Acknowledgment}

The authors extend their appreciation to the research center for advanced materials (RCAMS) at King Khalid University, Abha, Saudi Arabia for supporting this work through the research group program under grant number RCAMS/KKU/003/20.

\section{Author Contributions}

All authors contributed to data analysis, drafting or revising the article, have agreed on the journal to which the article will be submitted, gave final approval of the version to be published, and agreed to be accountable for all aspects of the work.

\section{Disclosure}

The authors report no conflicts of interest for this work and declare that there is no conflict of interest regarding the publication and results of this study.

\section{References}

1. Hay D, Hill M, Littlewood T. Neutropenia in primary care. $B M J$. 2020;349.

2. Hsieh MM. Prevalence of neutropenia in the U.S. population: age, sex, smoking status and ethnic differences. Ann Intern Med. 2007;146 (7):486-492. doi:10.7326/0003-4819-146-7-200704030-00004
3. Frater JL. How I investigate neutropenia. Int J Lab Hematol. 2020;42 (S1):121-132.

4. Haddy TB, Rana ARCO. Benign ethnic neutropenia: what is a normal absolute neutrophil count? J Lab Clin Med. 1999;133:15-22. doi:10.1053/lc.1999.v133.a94931

5. Lakhotia R, Aggarwal A, Link ME, Rodgers GP, Hsieh MM. Natural history of benign ethnic neutropenia in individuals of African ancestry. Blood Cells, Mol Dis. 2020;77:12-16. doi:10.1016/j.bcmd.2019.01.009

6. Paz MNEZ Z. The genetics of benign neutropenia. Isr Med Assoc J. 2011;13:625-629.

7. Denic S, Narchi H, Al Mekaini LA, Al-Hammadi S, Al Jabri ON, Souid AK. Prevalence of neutropenia in children by nationality. $B M C$ Hematol. 2020;16(1):1-7.

8. Gari M, Dakhakhni M, Gari A, et al. Incidence and potential causative factors associated with chronic benign neutropenia in the Kingdom of Saudi Arabia. BMC Proc. 2020;9(2):1-7.

9. León-Velarde F, Gamboa A, Chuquiza JA, Esteba WA, Rivera-Chira M, Monge CC. Hematological parameters in high altitude residents living at 4355, 4660, and 5500 meters above sea level. High Alt Med Biol. 2020;1(2):97-104. doi:10.1089/15270290050074233

10. Storz JF, Moriyama H. Mechanisms of hemoglobin adaptation to high altitude hypoxia. High Alt Med Biol. 2008;9(2):148-157. doi:10.1089/ham.2007.1079

11. Denic S, Showqi S, Klein C, Takala M, Nagelkerke N, Agarwal MM. Prevalence, phenotype and inheritance of benign neutropenia in Arabs. BMC Blood Disord. 2020;9(1):1-8.

12. Taha EH, Elshiekh M, Alborai A, et al. Normal range of white blood cells and differential count of Sudanese in Khartoum state. Int $J$ Adv Med. 2018;5(4):784. doi:10.18203/2349-3933.ijam20183116

13. Weingarten MA, Pottick-Schwartz EABA. The epidemiology of benign leukopenia in Yemenite Jews. Isr J Med Sci. 1993;29(5):297-299.

14. Carballo C, Foucar K, Swanson P, Papile LA, Watterberg KL. Effect of high altitude on neutrophil counts in newborn infants. $J$ Pediatr. 1991;119(3):464-466. doi:10.1016/S0022-3476(05)82065-7

15. Lambert RM, Baer VL, Wiedmeier SE, Henry E, Burnett J, Christensen RD. Isolated elevated blood neutrophil concentration at altitude does not require NICU admission if appropriate reference ranges are used. J Perinatol. 2020;29(12):822-825.

16. Wang J, Yu J, Fan J, He Y, Dong W, Wang Z. Evaluation of altitude-appropriate reference ranges for neutrophils in diagnosis of sepsis in very low birth weight infants: A multicenter retrospective study. PLoS One. 2017;12(2):e0171571. doi:10.1371/journal.pone.0171571

17. Siqués P, Brito J, León-Velarde F, et al. Hematological and lipid profile changes in sea-level natives after exposure to 3550-m altitude for 8 months. High Alt Med Biol. 2020;8 (4):286-295. doi:10.1089/ham.2007.8405

18. Peterson RF, Peterson WG. The differential count at high altitudes. J Lab Clin Med. 2020;20(7):723-726.

19. Luo X, Feng L, Bai X, et al. Continuous changes in biological levels of complete blood count in a high altitude area of China. SAGE Open Med. 2020;8]:205031212093133.

20. Palmblad J, Höglund P. Ethnic benign neutropenia: A phenomenon finds an explanation. Pediatr Blood Cancer. 2020;65(12):e27361.

21. Thobakgale CF, Ndung'u T. Neutrophil counts in persons of African origin. Curr Opin Hematol. 2020;21(1):50-57.

22. Chang EY, Tormey CA, Siddon AJ, Rahmani M, Wong EY. Duffy antigen phenotyping is a useful and clinically available test for benign ethnic neutropenia. Blood. 2018;132(Supplement 1):2546. doi:10.1182/blood-2018-99-117178

23. Chaitowitz M, Laura VM, Goel S. Hematology referrals for leukopenia in the bronx rarely result in clinically significant diagnoses: first steps towards and evidence-based referral guideline. Blood. 2016;128 (22):4743. doi:10.1182/blood.V128.22.4743.4743

24. Nagalapuram V, McCall D, Palabindela P, et al. Outcomes of isolated neutropenia referred to pediatric hematology-oncology clinic. Pediatrics. 2020;146:4. doi:10.1542/peds.2019-3637 
25. Mant M, Gordon PA, Akabutu J. Bone marrow granulocyte reserve in chronic benign idiopathic neutropenia. Clin Lab Haematol. 2020;9 (3):281-288.

26. Shoenfeld Y, Ben-Tal O, Berliner S, Pinkhas J. The outcome of bacterial infection in subjects with benign familial leukopenia (BFL). Biomed Pharmacother. 1985;39(1):23-26.

27. Palmblad J, Siersma V, Lind B, Bjerrum OW, Hasselbalch H, Andersen CL. Age-related prevalence and clinical significance of neutropenia - isolated or combined with other cytopenias: real world data from 373820 primary care individuals. Am J Hematol. 2020;95(5):521-528. doi:10.1002/ajh.25756
28. Boxer LA. How to approach neutropenia. Hematology 2010, the American Society of Hematology Education Program Book. 2012;1:174-182.

29. Gibson C, Berliner N. How we evaluate and treat neutropenia in adults. Blood. 2014;124(8):1251-1258. doi:10.1182/blood-2014-02482612

\section{Publish your work in this journal}

The International Journal of General Medicine is an international, peer-reviewed open-access journal that focuses on general and internal medicine, pathogenesis, epidemiology, diagnosis, monitoring and treatment protocols. The journal is characterized by the rapid reporting of reviews, original research and clinical studies across all disease areas. The manuscript management system is completely online and includes a very quick and fair peer-review system, which is all easy to use. Visit http://www.dovepress.com/ testimonials.php to read real quotes from published authors.

Submit your manuscript here: https://www.dovepress.com/international-journal-of-general-medicine-journal 